\title{
A TREE VERSION OF KÖNIG'S THEOREM
}

\author{
RON AHARONI, ELI BERGER, AND RAN ZIV
}

\begin{abstract}
König's theorem states that the covering number and the matching number of a bipartite graph are equal. We prove a generalisation, in which the point in one fixed side of the graph of each edge is replaced by a subtree of a given tree. The proof uses a recent extension of Hall's theorem to families of hypergraphs, by the first author and P. Haxell [3.
\end{abstract}

\section{WidTh AND MATChING WIDTH OF FAMILIES OF TREES}

A matching in a hypergraph is a set of disjoint edges. In [3] (see also [2] and 5) there were introduced a few notions which proved to be fruitful in the study of matchings. The two main ones are those of width and matching width, which are presented below.

Let $H$ and $F$ be two hypergraphs on the same vertex set. A subset $C$ of $F$ is said to be $H$-covering (or "an $H$-covering") if every edge in $H$ meets some edge from $C$ (in other words, the union of $C$ is a vertex cover for $H$ ). The $F$-width $w(H, F)$ of $H$ is the minimal size of an $H$-covering set of edges from $F$. The $F$-matching width $m w(H, F)$ of $H$ is the maximum, over all matchings $M$ in $H$, of $w(M, H)$. We write $w(H)$ and $m w(H)$ for $w(H, H)$ and $m w(H, H)$, respectively, and call these parameters the width and matching width of $H$. These notions have counterparts in which the covering set is required to be a matching. The independent $F$-width $i w(H, F)$ of $H$ is the minimal size of a covering matching in $F$. The independent matching width $\operatorname{imw}(H, F)$ of $H$ is the maximum, over all matchings $M$ in $H$, of $i w(M, F)$. Again, we write $i w(H)$ for $i w(H, H)$ and $i m w(H)$ for $i m w(H, H)$, and call them the independent width and independent matching width of $H$, respectively. (The notion of independent matching width is used in [3], but is not given there a special name.)

In [3] the following theorem was proved:

Theorem 1.1. If $\mathcal{A}$ is a family of hypergraphs such that $m w(\cup \mathcal{B}) \geq|\mathcal{B}|$ for every $\mathcal{B} \subseteq \mathcal{A}$, then there is a choice of disjoint edges, one from each hypergraph in $\mathcal{A}$.

Clearly, $m w \leq w$ and $i m w \leq i w$, and in general strict inequalities occur. However, as we shall prove below, for families of trees equality holds in both. In fact, both equalities are special cases of a more general result. For two hypergraphs $H_{1}, H_{2}$ and a symmetric relation $\sim$ on $H_{2}$ we denote by $w\left(H_{1}, H_{2}, \sim\right)$ the minimal size of a $\sim$-related subset of $H_{2}$ which is an $H_{1}$-covering. By $m w\left(H_{1}, H_{2}, \sim\right)$ we denote the maximum, over all matchings $M$ in $H_{1}$, of $w\left(M, H_{2}, \sim\right)$.

The research of the first author was supported by the fund for the promotion of research at the Technion. 
Theorem 1.2. Let $H_{1} \subseteq H_{2}$ be families of subtrees of a given tree. Let also be a symmetric relation on $\mathrm{H}_{2}$ containing the disjointness relation (i.e, if $x, y$ are disjoint members of $\mathrm{H}_{2}$ then $x \sim y$ ). Then

$$
m w\left(H_{1}, H_{2}, \sim\right)=w\left(H_{1}, H_{2}, \sim\right)
$$

The equality $m w(H)=w(H)$ for a family $H$ of trees is obtained upon taking $H_{1}=H_{2}=H$ and taking $\sim$ to be the total relation (i.e., $x \sim y$ for all $x, y \in H$ ). The equality $i m w=i w$ is obtained upon taking $H_{1}=H_{2}=H$ and $\sim$ the disjointness relation.

The theorem will clearly follow from the following lemma:

Lemma 1.3. Let $H_{1}, H_{2}$ and $\sim$ be as above, and let $c, d$ be two intersecting edges of $H_{1}$. Then one of $w\left(H_{1} \backslash\{c\}, H_{2}, \sim\right)$ or $w\left(H_{1} \backslash\{d\}, H_{2}, \sim\right)$ is equal to $w\left(H_{1}, H_{2}, \sim\right)$.

(Once the lemma is proved, the theorem follows by deleting edges from $H_{1}$ one by one, until a matching is obtained).

Proof of the Lemma Write $w=w\left(H_{1}, H_{2}, \sim\right)$. Suppose, for contradiction, that $w\left(H_{1} \backslash\{c\}, H_{2}, \sim\right)=w\left(H_{1} \backslash\{d\}, H_{2}, \sim\right)=w-1$. Let $C$ be a $\sim$-related covering of size $w-1$ of $H_{1} \backslash\{d\}$ and $D$ a $\sim$-related covering of size $w-1$ of $H_{1} \backslash\{c\}$. Clearly, $D$ does not meet $c$ and $C$ does not meet $d$. Let $S_{1}$ be the connected part of $T \backslash(V(c) \cap \bigcup\{V(t): t \in C\})$ containing $d$ and let $S_{2}=T \backslash V\left(S_{1}\right)$. (Here $T \backslash A$, where $A$ is a subset of $V(T)$, denotes the forest obtained by deleting the vertices of $A$ from $T$.)

Let $C_{i}=\left\{g \in C: g \subseteq S_{i}\right\}(i=1,2)$ and $D_{i}=\left\{g \in D: g \subseteq S_{1}\right\}(i=1,2)$. Let also $K=C_{1} \cup D_{2} \cup\{c\}$ and $L=C_{2} \cup D_{1}$.

Obviously, $K$ is an $H_{1}$-covering, since the only members of $H_{1}$ not covered by $C_{1}$ and $D_{2}$ are those which meet both $S_{1}$ and $S_{2}$, and these are covered by $c$. Since $D$ does not meet $c$, the tree $c$ is $\sim$-related to all members of $D_{2}$. Also, by the definition of $C_{1}$, no tree from $C_{1}$ meets $c$, and hence $c \sim x$ for every $x \in C_{1}$. Thus $K$ is $\sim$-related. A similar argument shows that also $L$ is a $\sim$-related $H_{1}$-covering. But $|K|+|L|=2 w-1$, and hence either $|K|<w$ or $|L|<w$, which contradicts the definition of $w$.

\section{A KÖNIG-LIKE THEOREM}

A hypergraph $H$ is called a point-tree hypergraph if it is obtained from a bipartite graph by replacing, in each edge, the point in one side of the graph (the same side for all edges) by a tree. More formally, $H$ is point-tree if, for some set $X$ and a tree $T$ whose vertex set is disjoint from $X$, each edge $e \in H$ is of the form $\{x\} \cup V(t)$, where $x=x(e) \in X$ and $t=t(e)$ is a subtree of $T$. For such a hypergraph we denote by $\sigma(H)$ the number $w(H, F)$, where $F=F(H)=\{\{x(e)\}: e \in H\} \cup\{V(t(e))$ : $e \in H\}$. (That is, the edges of $F$ are to be caught by vertices in $X$, or trees $t(e)$ ). Recall that the matching number $\nu(H)$ of a hypergraph $H$ is the maximal size of a matching in $H$. We shall prove the following generalisation of König's theorem:

Theorem 2.1. $\sigma(H) \leq \nu(H)$ for every point-tree hypergraph $H$.

König's theorem is the special case in which all trees $t(e)$ are singletons.

In [6] (see also [4]) there were considered objects which are similar to point-tree hypergraphs: bipartite graphs in which each point (on both sides of the graph) in each edge is replaced by an interval. In [6] it was proved that $\tau \leq 2 \nu$ for such 
hypergraphs. It is interesting to note that the proof there was topological (as was the simpler proof in [4]), and at base so is the proof here, since it relies on Theorem 1.1. whose proof is topological.

In fact, we shall need a stronger version of Theorem 1.1, its "deficiency version".

Definition 2.2. The deficiency $\operatorname{def}(\mathcal{A})$ of a family $\mathcal{A}$ of hypergraphs is the minimal natural number $d$ such that $m w(\bigcup \mathcal{B}) \geq|\mathcal{B}|-d$ for every subfamily $\mathcal{B}$ of $\mathcal{A}$.

The following strengthening of Theorem 1.1 follows from it by a standard argument (see [1]).

Theorem 2.3. Every family $\mathcal{A}$ of hypergraphs has a subfamily $\mathcal{D}$ of size at most $\operatorname{def}(\mathcal{A})$, such that $\mathcal{A} \backslash \mathcal{D}$ has a choice function of disjoint edges.

Proof of Theorem 2.1 For each $x \in X$ let $K(x)$ be the hypergraph $\{V(t(e))$ : $x(e)=x\}$. Applying Theorem 2.3 to the family $\{K(x): x \in X\}$ yields the existence of a subset $Y$ of $X$ such that, writing $K=\bigcup\{K(y): y \in Y\}$, we have $m w(K)=|Y|-(|X|-\nu(H))$. By Lemma 3.1 $w(K)=m w(K)$. Let $C$ be a $K$ covering set of edges in $K$ of size $|Y|-(|X|-\nu(H))$. Then $C \cup X \backslash Y$ is an $H$-covering set consisting of elements of $F(H)$, of size $\nu(H)$. This proves the theorem.

\section{The SPECIAL CASE OF FAMILIES OF INTERVALS}

A special case of trees is that of intervals. Thus, all the results above apply to the special case of families of intervals. But it turns out that in this case the equalities $m w=w$ and $i m w=i w$ can be strengthened. For example, the matchings which are "hard to catch" can be described explicitly, and can be found efficiently. To show this, we shall need the following notation.

We say that a set of vertices in a graph $G$ is $k$-independent if the distance between any two of its members is larger than $k$. (Thus 1-independence is just the usual independence.) By $\gamma_{k}(G)$ we denote the maximal size of a $k$-independent set in $G$. A set of edges in a hypergraph $H$ is $k$-remote if it is $k$-independent in the line graph $L(H)$. We write $\zeta_{k}(H)$ for $\gamma_{k}(L(H))$. Clearly, for all hypergraphs we have

$$
\zeta_{2} \leq m w \leq w
$$

Here we shall prove:

Lemma 3.1. For a family $F$ of intervals on the real line equality holds throughout (1), namely $\zeta_{2}(F)=m w(F)=w(F)$.

Given a family of intervals $F$, we denote by $\ell(F)$ the leftmost interval in $F$, namely the one with minimal right endpoint. For a real number $x$ we denote by $F(>x)$ the set of those intervals in $F$ which lie entirely within $(x, \infty)$.

Proof of Lemma 3.1 By (11) the lemma will be proved if we can exhibit a 2-remote set $R$ and a covering set $C$ of the same size. We construct such sets by an inductive process.

Let $r_{1}=\ell(F)$, and $c_{1}=\left[x_{1}, y_{1}\right]$ an element of $F$ meeting $r_{1}$ whose right endpoint is largest. Let now $r_{2}=\ell\left(F\left(>y_{1}\right)\right)$, and $c_{2}=\left[x_{2}, y_{2}\right]$ the interval in $F$ meeting $r_{2}$ whose right endpoint is maximal. (Note that $c_{2}$ may intersect $c_{1}$.) In general, having defined $c_{i}=\left[x_{i}, y_{i}\right](i>0)$, we let $r_{i+1}=\ell\left(F\left(>y_{i}\right)\right)$ and choose $c_{i+1}$ as 
the interval in $F$ meeting $r_{i+1}$ and extending furthest to the right. The process terminates when, at some stage $t$, there is no interval lying entirely to the right of $c_{t}$. Then, clearly, $R=\left\{r_{1}, \ldots, r_{t}\right\}$ is remote, while $C=\left\{c_{1}, \ldots, c_{t}\right\}$ is covering.

A similar equality can be proved for $\zeta_{k}$ for all $k$. For any graph $G$ we denote by $\rho_{k}(G)$ the minimal number of vertex sets of diameter at most $k$, whose union is $V(G)$. Obviously, $\rho_{k}(G) \geq \gamma_{k}(G)$ for all $k$. One corollary of Lemma 3.1 is that in an interval graph $\rho_{2}(G)=\gamma_{2}(G)$ (in fact, it implies something even stronger, namely that an interval graph $G$ can be partitioned into $\gamma_{2}(G)$ sets of radius 1.) This equality is true for all $k$, that is, in an interval graph $\rho_{k}(G)=\gamma_{k}(G)$ for all $k$. We shall prove this for a more general class of graphs, that of incomparability graphs. A graph is called an incomparability graph if there exists a partial order on its vertex set, such that two vertices in the graph are connected if and only if they are incomparable. As is well known, an interval graph is an incomparability graph, the order on the intervals being that of "lying completely to the right of".

Proposition 3.2. In an incomparability graph $\rho_{k}(G)=\gamma_{k}(G)$ for all $k$.

Given a graph $G$ and a natural number $k$, we denote by $G^{* k}$ the graph with the same vertex set as $G$, in which two vertices are connected if and only if their distance in $G$ does not exceed $k$. Obviously, $\gamma_{k}(G)=\gamma\left(G^{* k}\right)$, and $\rho_{k}(G)=\rho\left(G^{* k}\right)$. Thus, if $G^{* k}$ is perfect, then $G$ satisfies Proposition 3.2. Since incomparability graphs are known to be perfect, Proposition 3.2 will follow from:

Lemma 3.3. If $G$ is an incomparability graph then so is $G^{* k}$.

Proof Let " $\succ$ " be a partial order on $V(G)$ such that $x$ and $y$ are connected in $G$ if and only if they are $\succ$-incomparable. Let " $\sqsupset$ " be the relation defined by $x \sqsupset y$ if and only if $x \succ y$ and $(x, y) \notin E\left(G^{* k}\right)$. Clearly, it suffices to show that " $\sqsupset$ " is a partial order.

Assume that it is not. Then there exist $x, y, z \in V(G)$ such that $x \sqsupset y, y \sqsupset z$, while $x \nrightarrow z$. There exist then vertices $x=u_{0}, u_{1}, u_{2}, \ldots, u_{t}=z(t \leq k)$ such that $u_{i}$ is $\succ$-incomparable to $u_{i+1}$ for all $0 \leq i<t$. Since $x \sqsupset y$, all $u_{i}$ must be $\succ$-comparable to $y$ (or else the sequence $u_{0}, \ldots, u_{i}, y$ would show that $(x, y) \in E\left(G^{* k}\right)$ ). Since $u_{0} \succ y$ and $y \succ u_{t}$, it follows that there exists $i<t$ such that $u_{i} \succ y$ and $y \succ u_{i+1}$. But then, by the transitivity of the relation $\succ$, we have $u_{i} \succ u_{i+1}$, a contradiction.

Our next observation on the interval case is that the equality $i m w=i w$ can be given in this case a constructive (and algorithmically efficient) proof.

Theorem 3.4. $i m w(F)=i w(F)$ for every hypergraph $F$ of intervals. Moreover, there is a polynomial time algorithm producing a matching $M$ such that iw $(M, F)=$ $i w(F)$.

Proof Write $k=i w(F)$. We shall prove the theorem by constructing a matching $M$ in $F$ satisfying:

(P) Every matching of size $k-1$ in $F$ misses an interval from $M$.

Note, first, that we can assume that no two endpoints of intervals in $F$ coincide: if there is such coincidence, we can enlarge some intervals, without changing the intersection pattern of the family. Henceforth we shall make this assumption.

For a matching $E$ in $F$ we write $E=\left(e_{1}, \ldots, e_{t}\right)$ if $e_{i}$ are the edges of $E$ ordered from left to right. Let $E$ be such a matching, and write $e_{j}=\left[a_{j}, b_{j}\right], j \leq t$. For 
each $j \leq t$ we denote by $d_{j}(E)$ the interval $\ell\left(F\left(>b_{j-1}\right)\right.$ ) (where $b_{0}$ is defined as $-\infty$, meaning that $d_{1}(E)$ is always $\left.\ell(F)\right)$. If $F\left(>b_{t}\right)$ is non-empty, we write also $d_{t+1}(E)=\ell\left(F\left(>b_{t}\right)\right)$. Note that $d_{j}(E)$ meets $e_{j}$ if and only if there is no interval from $F$ lying between $e_{j-1}$ and $e_{j}$; or, in the case $j=1$, if and only if there is no interval in $F$ lying to the left of $e_{1}$. By $m(E)$ we denote the maximal index $m \leq t$ such that there is no interval from $F$ lying between $e_{j-1}$ and $e_{j}$ for all $j \leq m$ (if there is an interval from $F$ to the left of $e_{1}$, we write $m(E)=1$ ). If $m(E)=t$, we say that $E$ is dense. Note that if $E$ misses some edge from $F$ then it misses $d_{m(E)}(E)$.

Let $L_{i}$ be the set of all right endpoints of intervals $e_{i}$ in some dense matching $E=\left(e_{1}, \ldots, e_{t}\right)$. An interval $[a, b]$ is called $L_{i}$-free if $[a, b] \cap L_{i} \subseteq\{b\}$. Let $M$ be the set of all intervals in $F$ which are equal to $d_{i}(E)$ for some dense matching $E$, and are $L_{j}$-free for all $j \leq i$.

The proof will be complete if we show that $M$ is a matching, and that it satisfies property $(\mathrm{P})$.

Let us first show that $M$ is a matching. Suppose, for contradiction, that $d=$ $d_{p}(E)=[a, b]$ and $d^{\prime}=d_{q}\left(E^{\prime}\right)=\left[a^{\prime}, b^{\prime}\right]$ are two intersecting members of $M$, where $E=\left(e_{1}, \ldots, e_{p-1}\right)$ and $E^{\prime}=\left(e_{1}^{\prime}, \ldots, e_{q-1}^{\prime}\right)$ are two dense matchings. By assumption $b \neq b^{\prime}$, so we may assume (say) that $b<b^{\prime}$. Note that $b \in L_{p}$, since the matching $\left(e_{1}, \ldots e_{p-1}, d\right)$ is dense. Since $d^{\prime} \in M$, this implies that $q<p$. Let $y$ be the right endpoint of $e_{q-1}^{\prime}$. Since $d \in M$ and $y \in L_{p-1}$, it follows that $y<a$. But then the interval $d=[a, b]$ witnesses the fact that $d^{\prime} \neq \ell(F(>y))$, contradicting the definition of $d^{\prime}$.

Next we show that $M$ satisfies property (P). Suppose that there exists a matching $Z=\left(z_{j}: j \leq t\right)$ in $F$ meeting all intervals in $M$, where $t<k$. Among all such matchings $Z$, choose one in which the right endpoint of $z_{m(Z)}$ is maximal. Write $m=m(Z)$ for this $Z$. Since $i w(F)=k$, the matching $Z$ misses an interval from $F$. This means that $Z$ misses $d=d_{m}(Z)$. If $d \in M$, we are done. If not, then $d$ contains a point from $L_{j}$ for some $j \leq m$. That is, it is the right endpoint of an interval $w_{j}$ in some dense matching $W=\left(w_{1}, \ldots, w_{m}\right)$. But then the matching $\left(w_{1}, \ldots, w_{j}, z_{m+1}, \ldots, z_{t}\right)$, like $Z$, does not miss any edge from $M$. This contradicts the maximality property of $Z$.

To see that $M$ above can be constructed in polynomial time, it suffices to note that the sets $D_{j}$ of those intervals which appear as the $j$-th element in some dense matching can be constructed inductively in plynomial time, and that $M$ is defined by these sets.

\section{REFERENCES}

[1] R. Aharoni, Ryser's conjecture for tripartite hypergraphs, Combinatorica, to appear

[2] R. Aharoni and M. Chudnovsky, Special triangulations of the simplex and systems of disjoint representatives, submitted for publication

[3] R. Aharoni and P. Haxell, Hall's theorem for hypergraphs, Jour. Graph Th., to appear

[4] T. Kaiser, Transversals of $d$-intervals, Discrete. Comput. Geom. 18 (1997), 195-203.

[5] R. Meshulam, The clique complex and hypergraph matching, Combinatorica, to appear

[6] G. Tardos, Transversals of 2-intervals, a topological approach, Combinatorica 15 (1995), 123134. 
Department of Mathematics, Technion, Haifa, Israel 32000

E-mail address, Ron Aharoni: ra@tx.technion.ac.il

E-mail address, Eli Berger: beli@ibm.il.com

Department of Computer Science, Tel-Hai college, Upper Galilee, Israel 12210

E-mail address, Ran Ziv: ranziv@telhai.ac.il 